\title{
The attribute selection process in pattern perception: The effect of constraint redundancy and stimulus exposure time on the classification of spatially represented Markov patterns*
}

\author{
DONALD F. DANSEREAU \\ Texas Christian University, Fort Worth, Texas 76129 \\ and \\ BILL R. BROWN \\ University of Louisville, Louisville, Kentucky 40208
}

\begin{abstract}
A transfer paradigm was used to study the effect of constraint redundancy $(\mathrm{Rc})$ and stimulus exposure time (ET) on the "same-different" classifications of pairs of spatially represented Markov patterns (Vargus 7 stimuli; Evans \& Mueller, 1966). In the training phase, each S classified, without feedback, pairs of Vargus 7 stimuli generated as deviations from three prototypes (i.e., most probable sequences). Each $S$ received stimuli generated at one of three levels of Rc and exposed for one of three durations. In the test phase, all Ss classified different deviations from the same three prototypes at a single Rc level and a single ET level. Unsupervised classification performance was above chance in both training and transfer and increased slightly over trials. Significant Rc and ET effects were found. These results supported two models of attribute selection and utilization; discussions of these models and their relationships to the findings were directed toward the analysis of the "schema plus correction" hypothesis.
\end{abstract}

A number of researchers concerned with the encoding of patterned stimuli have suggested that families of visual patterns (i.e., instances that are related to one another by a number of common attributes) are stored in a structure that relates each individual stimulus to a representation of the communalities occurring within the entire family. For example, a particular dog may be stored as a set of deviations from the attributes which are common to all dogs. Such terms as schema plus correction (Woodworth, 1938; Oldfield, 1954), ideal plus transformation (Bregman, 1968), and central tendency (or prototype) plus distortion (Posner, 1968) have been used to describe this hypothesized storage configuration.

Encoding of schematic characteristics plus the deviations of individual stimuli from the schema represents an effective process for reducing memory storage (Oldfield, 1954; Attneave, 1957). The natural environment, however, seldom provides an intact, undistorted prototype or schema. To complicate matters, the members of several different but closely related schema families generally occur together. Such storage configurations as schema plus correction are thus relevant to ordinary human pattern perception and storage only if: (1) Ss are able to select the appropriate set of attributes that are critical for family membership (attribute selection), (2) Ss are able to encode the

*This research was supported by the Department of Defense, Project THEMIS Contract (DAAD05-68-C-0176), under the Department of Army to the Institute for the Study of Cognitive Systems through the TCU Research Foundation. Further reproduction is authorized to satisfy needs of the U.S. Government. attribute values that define the prototype (prototype abstraction), and (3) Ss are able to use the stored prototypes to classify and encode new distortions and to recognize and recall previously stored instances (prototype utilization).

A number of experiments have attempted to deal directly with prototype abstraction and utilization. Posner and Keele $(1968,1970)$ have shown that the prototype can be classified more efficiently than other instances of the same family after training on nonprototype family members. Studies by Hinsey (1963), Vinikoor (1968), and Norcross (1968) have shown that classification of distorted stimuli is enhanced by previous exposure to the prototype from which these peripheral instances are derived. There are, however, two factors that limit the generalizability of these studies to real world pattern perception: (1) the presence of feedback, and (2) the simplicity of the distortions used to produce schema families. Generally, feedback (knowledge of results) is provided to Ss in these studies to guide their abstraction and/or utilization processes. In the one case feedback was not employed, the Es, Posner and Keele (1970), obtained results which did not conform to previous findings. Another important factor, affecting generalizability, is that the schema families used experimentally have been produced primarily by applying simple, independent manipulations of prototype points, or by simple rotation. These conditions do not adequately simulate the natural environment in which feedback is usually not provided, and the available stimuli are distorted in a more complex fashion.

Experiments dealing with attribute selection are based 
on the fact that stimuli presented for classifications often vary along a large number of attributes or dimensions, only a subset of which determine family membership. The first step in learning a new schema family involves the selection of appropriate attributes through experience with the stimuli. This increased sensitivity to critical attributes is followed by the abstraction process, which involves the storage of attribute values defining the schema or prototype. Prototype utilization then follows directly. It should not be assumed, of course, that these processes necessarily occur sequentially; the processes undoubtedly overlap.

Studies in the area of attribute selection have been designed to explore the variables controlling the selection process. To date, it has been shown that: (1) Ss can become increasingly sensitive to the attributes that define schema families without the presence of external feedback (Hastings et al, 1969; Hastings, 1970; Harris et al, 1970; and Rankin \& Evans, 1968). It should be noted, however, that the families used in these studies are formed by producing simple, attribute-independent (dependent only on spatial location of the attribute) deviations from the prototypes. (2) The amount of deviation strongly influences performance on tasks requiring sensitivity to an appropriate set of attributes (Brown et al, 1968; Brown \& Dansereau, 1969).

There are, however, still a number of issues relating to attribute selection that must be resolved in order to provide the groundwork for further experimentation on prototype abstraction and utilization:

(1) Can Ss become increasingly sensitive to an appropriate set of attributes, without external feedback, when the schema families are produced by complex distortions from a prototype? An example of such stimuli would be those created by mapping the results of 2. Markov process into the spatial domain. Patterns of this type would be analogous to graphical representations of time series data (e.g., EEG records). In addition, deviations from the most probable Markov sequence can represent complex rotations of parts of a spatial pattern. Studies involving spatially represented Markov stimuli have found that Ss were influenced by the attributes (Brown \& Dansereau, 1969; Brown \& Evans, 1969), but have not found an improvement in classification performance over trials. It is, therefore, not clear that $\mathrm{Ss}$ can learn to refine their attribute selections in the face of more complex distortion.

(2) Do Ss become sensitive to the level and type of stimulus distortion as well as to the relevant attributes for schema discrimination? This question stems from Attneave's (1957) hypothesis that Ss learn the relative variability of the family members about the prototype as well as the schema or prototype itself. If this notion is correct, transferring Ss from one level of distortion to another should strongly affect certain aspects of their pattern classification performance. Specifically, in a task requiring the $S$ to say whether two patterns belong to the same family or to different families, it might be expected that the threshold for making "same" or "different" judgments would be altered following training on stimuli at various distortion levels.

(3) Does Ss' performance vary systematically with stimulus exposure time? The relationship of exposure time to performance may depend heavily on the processes Ss employ in making classification judgments; therefore, specification of the relationship may lead to more detailed hypotheses about attribute selection.

The present experiment was designed to explore the issues cited above. Ss were required to classify spatially represented Markov patterns without feedback (knowledge of results). In the training phase, amount of distortion (three levels) and exposure time (three levels) were treated as between-S variables. In the test phase, all Ss served under a single distortion level and a single stimulus exposure time.

Since the study involved multiple independent variables and was primarily exploratory in nature, detailed hypotheses were not made. In general, it was expected that increasing the distortion of the training stimuli from the prototype would be deleterious to performance in both the training and test phases. The effect of increasing stimulus exposure time was expected to improve performance, but interactions of this variable with distortion level were anticipated.

\section{METHOD}

\section{Subjects}

The Ss consisted of 90 undergraduates enrolled in introductory psychology courses at Texas Christian University.

\section{Stimuli}

The Vargus 7 computer program (Evans, 1967a) was used to generate Markov digit strings of $50 \%, 70 \%$, and $100 \%$ constraint redundancy (Rc). The Rc measure has been discussed in detail by Evans (1967b) and refers to the extent to which the stimuli adhere to a schema rule (i.e., the average degree of distortion over the entire family of patterns). The $100 \% \mathrm{Rc}$ strings are simply cyclic permutations of one another (e.g., 1, 2, 3, 4;2, 3, 4,$1 ; 3,4,1,2$ would all be $100 \%$ Rc members of the same family). In a sense, these stimuli are examples of the Markov prototype (i.e., the most probable sequence). Strings produced at the $70 \% \mathrm{Rc}$ level have the same cyclic permutation properties, but they also contain deviations from the most probable sequence (transition probabilities along the most probable sequence are .88 in this case). At $50 \% \mathrm{Rc}$ the stimuli have an even higher probability of being distorted (.79 is the probability of a most probable sequence transition).

These sets of digit strings, produced by the Vargus 7 program, were mapped into histoform (histogram-like) patterns for spatial presentation. The instances were 24 columns in length and were produced from a seven-element Markov process by converting the elements into column heights. The program selected column heights with transitional probabilities favoring three different most probable sequences (MPSs). The stimulus populations sampled in the present study can be found in Bersted, Brown, and Evans (1968a), and are identified as Schemata 2, 3, and 4.

The manner in which the stimuli were generated assured that corresponding instances of the three schema families had the same variance. This variance measure is the proportion of schematic steps (POSS) for each instance, and it describes the 
adherence of each individual instance to the MPS. The POSS statistic has been discussed in detail by Bersted et al (1968a). The computed POSS values for stimuli generated at $100 \% \mathrm{Rc}$ would, of course, always be 1.00 , although the first column in each of these instances could correspond to any of the seven elements in the MPS.

\section{Training Task}

The Ss were given 60 trials with $50 \%, 70 \%$, or $100 \%$ Vargus 7 patterns. On a typical trial, the Ss viewed two stimuli and then judged them to be of the same family or of different families. The 60 trials consisted of 30 pairings of instances from different schema families and 30 pairings from the same family.

A Kodak Carousel projector, impulsed by tapes that controlled the various timing sequences, projected each of the 60 stimulus pairs onto a $2 \times 2 \mathrm{ft}$ while rear projection screen. For each of the three $\mathrm{Rc}$ conditions, the following durations constituted the presentation time variable: (1) $2 \sec (100 \%-2$, $70 \%-2$, and $50 \%-2$ groups); (2) $5 \sec (100 \%-5,70 \%-5$, and $50 \%-5$ groups); and (3) $8 \mathrm{sec}(100 \%-8,70 \%-8$, and 50\%-8 groups). The Ss were randomly assigned to the nine treatment conditions (10 Ss per group). Groups of two Ss, seated $5 \mathrm{ft}$ from the screen, were given $7 \mathrm{sec}$ to mark their judgments of each pair on an answer sheet. A variable intertrial interval was used to equate all Ss with respect to the total amount of time spent in the task.

No one instance from any of the three schemata was presented more than once during the 60 trials. The two instances presented on each trial, whether they were from the same or different schemata, were paired on the basis of their having equal POSS values. This procedure assured that the instances of any given pair were equal in difficulty as far as abstraction of the schema rules was concerned. In the case of the $100 \% \mathrm{Rc}$ stimuli, the patterns were paired on the basis of their first columns being of different heights. Two stimulus presentation orders were used. These were randomly produced under the constraint that each block of 10 trials contained an equal number of "same" and "different" pairings.

\section{Test Task}

Following training, all Ss received 60 trials of $70 \%$ Rc patterns sampled from the same three schema families used in the training task; however, no stimulus appeared in both the training and test phase. A 5-sec presentation time and 7-sec response time were used for all Ss. As in training, $50 \%$ of the 60 trials involved the pairing of instances from different schema families; the remaining $50 \%$ of the trials consisted of pairs of instances sampled from the same family. Instances from the three schema families were represented only once in the task. Two presentation orders were obtained, using exactly the same method as used in the training phase. These orders were equally represented within the nine training conditions

\section{RESULTS}

In order to evaluate the effects of the two stimulus presentation orders upon performance, a three-factor analysis of variance (with Rc, exposure time, and presentation order as variables) was conducted separately for the training and test task. Rc and exposure time (ET) for the test groups were the conditions they were exposed to during training. The number of correct same-different judgments made across 60 trials served as the dependent measure for each of these analyses. The order of stimulus presentation, in both the training and testing phases, was not significant

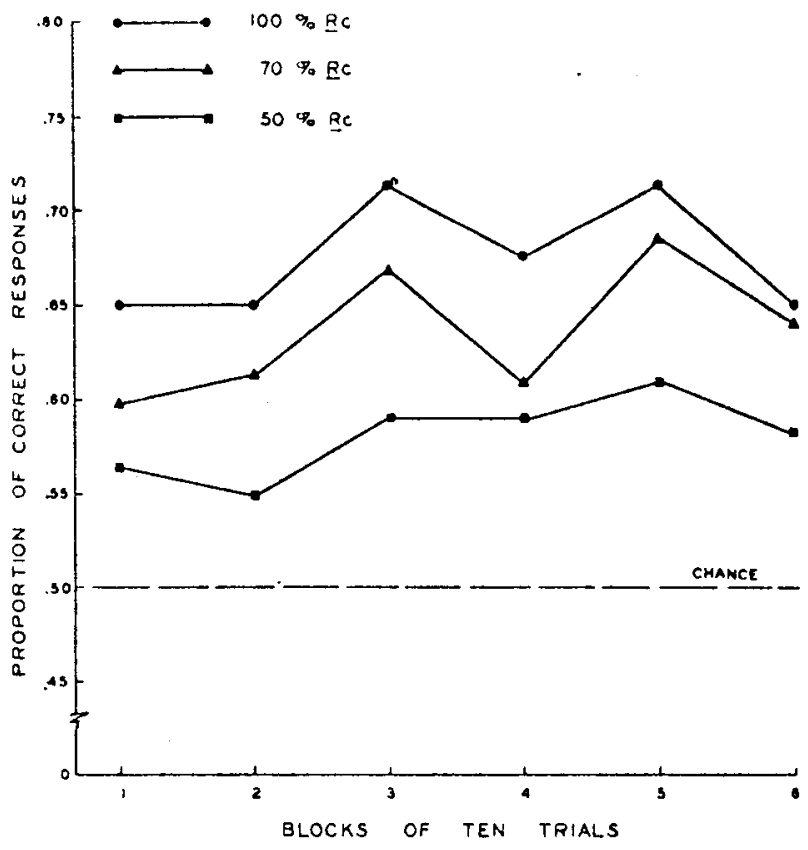

Fig. 1. Proportion of correct "same-different" responses in training for the three Rc groups as a function of blocks of trials.

and did not significantly interact with the Rc or ET conditions present in the training task. In order to simplify the subsequent analyses, the two presentation orders were combined for all treatment groups.

\section{Training Task}

A three-way analysis of variance with two between-group factors ( $R c$ and $E T$ ) and one within-group factor (blocks of 10 trials) was performed with the number of correct same-different judgments made in the training task as the dependent variable. There was a significant blocks effect, $F(5,405)=7.84$, $p<.001$, a significant $R c$ main effect, $F(2,81)=13.61$, $\mathrm{p}<.001$, and a significant ET main effect, $\mathrm{F}(2,81)=$ $7.83, \mathrm{p}<.01$. Figure 1 shows that increasing the magnitude of $\mathrm{Rc}$ on the training task facilitated performance. Performance also improved as a function of increasing the amount of ET (see Fig. 2). The Rc by ET interaction was also significant, $F(4,81)=4.77$, $\mathrm{p}<.05$; a highly significant portion of the variance accounted for by this interaction may be attributed to the simple main effect of ET at the $100 \% \mathrm{Rc}$ level, $F(2,81)=100.62, p<.001$. Figure 3 shows that increasing ET facilitated performance under the $100 \%$ $\mathrm{Rc}$ condition, but produced little effect on performance under the $50 \%$ and $70 \% \mathrm{Rc}$ conditions. The simple effects of ET at these latter two Rc levels were not significant.

\section{Test Task}

A three-way analysis of variance with two between-group factors ( $\mathrm{Rc}$ and ET training conditions) 


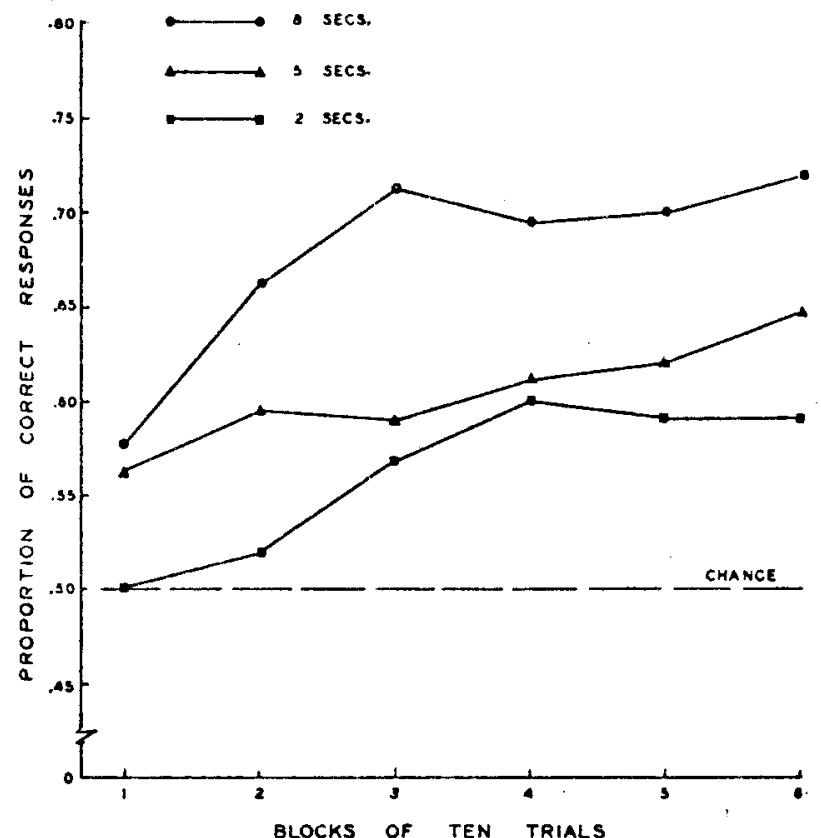

Fig. 2. Proportion of correct "same-different" responses in training for the three ET groups as a function of blocks of trials.

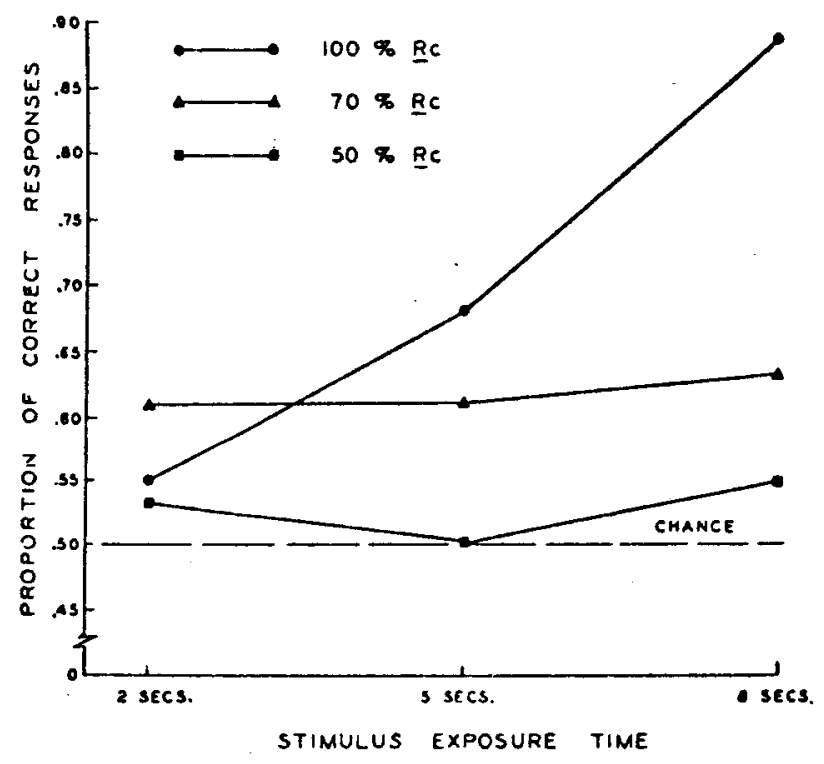

Fig. 3. Proportion of correct "same-different" responses in training for the groups serving under the various Rc-ET combinations.

and one within-group factor (blocks of five trials) was used to analyze performance in the test task. The number of correct same-different judgments made in the testing phase constituted the dependent measure. As in training, the blocks effect was significant, $F(5,405)=$ $3.40, \mathrm{p}<.01$. Moreover, the $\mathrm{Rc}$ main effect was significant, $F(2,81)=4.98, p<.01$. Inspection of Fig. 4 shows that performance in the test task was facilitated by increasing the magnitude of $\mathrm{Rc}$ present in training.

The above analysis was accompanied by a $t$ test for independent groups in order to compare the mean correct judgments made in the test task by the $50 \% \mathrm{Rc}$ training group (collapsed across ET) to that exhibited in training under the $70 \% \mathrm{Rc}$ condition (collapsed across ET). Contrary to expectation, the performance mean for the $70 \% \mathrm{Rc}$ group in training was not significantly different from that of the $50 \% \mathrm{Rc}$ training group in the test task. Training on $50 \% \mathrm{Rc}$ stimuli thus did not facilitate performance with $70 \% \mathrm{Rc}$ stimuli in comparison to a no-training condition.

In order to provide more detailed information about the effects of shifts in Rc magnitude on performance, a three-way analysis of variance, with two between-group factors ( $\mathrm{Rc}$ and ET) and one within-group factor (training task and test task), was conducted using the total number of "different" judgments (trials on which Ss judged the patterns to be from different families) as the dependent variable. The $\mathrm{Rc}$ by Task interaction was highly significant, $F(2,81)=26.10, p<.001$. Table 1 shows that increasing the magnitude of $\mathrm{Rc}$ tended in

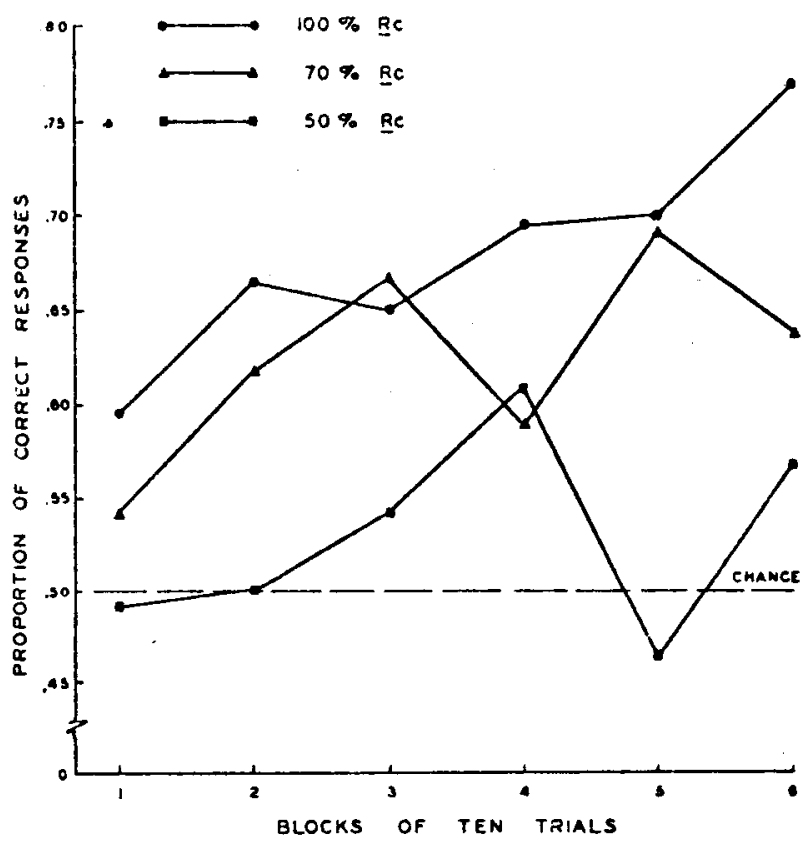

Fig. 4. Proportion of correct "same-different" responses in testing for the three Rc groups (in training) as a function of blocks of trials.

Table 1

Total Number of "Different" Responses: Correct and Incorrect"

\begin{tabular}{ccc}
\hline Groups & Training & Transfer \\
\hline $100-8$ & 315 & 375 \\
$100-5$ & 330 & 388 \\
$100-2$ & 357 & 420 \\
$70-8$ & 367 & 347 \\
$70-5$ & 393 & 364 \\
$70-2$ & 382 & 389 \\
$50-8$ & 380 & 344 \\
$50-5$ & 391 & 326 \\
$50-2$ & 392 & 384 \\
\hline
\end{tabular}

"There are 300 actual "different" trials for each of the nine groups. 
general to produce a decrement in the number of "different" responses in training. On the other hand, the number of such responses exhibited in testing became larger as a function of increased magnitudes of $\mathrm{Rc}$ present in the training phase. It is important to note that in all cases, the percentages of "different responses" were above the ideal $50 \%$ level. Although it might be expected that classification performance would improve as the "different" percentage approached $50 \%$, the opposite relationship was found in the test task.

\section{DISCUSSION}

The results of the training phase show that performance on the "same-different" discrimination task is above chance and increases slightly over blocks. These results clearly indicate that, even without knowledge of results, Ss can select an appropriate set of attributes upon which to base discrimination judgments of spatially represented Markov stimuli. Since the Markov process generates stimuli composed of "parts" (in this case, column heights) that depend only on their immediate predecessors and not on spatial position, it is apparent that Ss can learn to attend to relationships between stimulus "parts" independent of their spatial locations. With place-dependent stimuli, such as those used in the majority of abstraction and utilization studies (e.g., Vargus 9 stimuli, Evans \& Mueller, 1966), "parts" of patterns are correlated with both the preceding "parts" and with their spatial position. It is therefore not possible to determine which aspect of the pattern is critical to the Ss' judgments. The results of this experiment provide an initial step in specifying the critical features of attribute selection; it is apparent that Ss, at least under some circumstances, can utilize information based on local (first-order) relationships between the "parts" of spatial patterns. It remains to be determined to what extent this property is utilized when position information is also present.

There are at least two hypotheses about how Ss may be using the selected attributes in arriving at their "same-different" judgments. Upon presentation of two patterns to be judged same or different, the $S$ may, in some sense, "measure" the distance between the two stimuli along a set of attributes he has selected and then respond according to the magnitude of this difference (i.e., if small differences, respond "same"; if large differences, respond "different"). These "measurements" could be made without any reference to a schema or prototype. A second possibility is that each of the two stimuli is compared with each of the stored prototypes. The stimulus is then considered to be a distortion of the closest prototype. The stimuli are judged to be same if they are distortions of the same prototype and different if they are distortions of different prototypes.

Both of these hypotheses predict the results of the training task, including the apparent increase in the bias to say "different" as Rc decreases (Table 1). The effect of decreasing $\mathrm{Rc}$, due to the distributional properties of the sample (stimuli at small distance from the prototype occur more frequently than stimuli at large distances), is to increase the average distance along an appropriate set of attributes between stimuli of the same schema family and, by definition, to increase the average distances of stimuli from their prototype. These correlated increases would both result in a greater number of "different" responses and potentially poorer performance, depending on the thresholding operation.

The ET effect is also compatible with both hypotheses. Increased ET at $100 \% \mathrm{Rc}$ allows Ss to develop more accurate "measures" whether between stimuli or between the stimuli and their prototypes. At lower $\mathrm{Rc}(50 \%$ and $70 \%)$, the increased distances between pairs of stimuli and between the stimuli and their prototypes prohibit greater accuracy with greater exposure time.

The Rc effect in the analysis of correct judgments in the test task indicates that experience on the unsupervised discrimination of $100 \% \mathrm{Rc}$ stimuli, which in effect are Markov prototypes, is more effective than identical experience with distortions of these prototypes, even when the level of distortion in training is the same as with the test stimuli (70\% to $70 \%$ condition). These results are in accord with Attneave (1957) and Hinsey (1963), who found facilitation of performance on family members after exposure to the prototypes. The results also confirm the findings of Lawrence (1952), Baker and Osgood (1954), and Restle (1955). These investigators have shown that, under certain circumstances, a difficult discrimination may be learned more easily if the Ss are trained on an easy discrimination of the same type than if all training is given directly on the difficult discrimination.

The fact that prior familiarization with the prototypes facilitated discrimination of peripheral stimuli does illustrate what has been termed "prototype utilization." In this case, however, such a conceptualization may be misleading in that the enhancement could be produced by an increase in sensitivity to the appropriate attribute space, as well as by the actual utilization of the stored prototypes. All that can be concluded, therefore, is that some information about the appropriate dimensions was stored during exposure to prototypical instances.

The significant result of the $t$ test comparing S-D performance on the $70 \% \mathrm{Rc}$ stimuli in training with performance on the $70 \% \mathrm{Rc}$ stimuli in the test phase after $50 \% \mathrm{Rc}$ training shows that, apparently, training on $50 \% \mathrm{Rc}$ stimuli is not of any positive value to the Ss in performing $70 \% \mathrm{Rc}$ discriminations in the test task. This result is supported by Bersted et al (1968b); they found that training on $50 \%$ Rc task did not, in general, improve subsequent performance on a reproduction task in comparison with a group pretrained on random patterns. It is suggested that individuals in the $50 \% \mathrm{Rc}$ group in training are not attending to an appropriate set of 
Table 2

Variances of the Total Scores

\begin{tabular}{ccc}
\hline Groups & Training & Transfer \\
\hline $100-8$ & 77.8 & 35.8 \\
$100-5$ & 126.9 & 50.9 \\
$100-2$ & 45.4 & 59.1 \\
$70-8$ & 38.1 & 55.9 \\
$70-5$ & 46.8 & 92.2 \\
$70-2$ & 6.0 & 39.1 \\
$50-8$ & 13.3 & 32.1 \\
$50-5$ & 4.7 & 23.9 \\
$50-2$ & 5.8 & 8.0 \\
\hline
\end{tabular}

attributes, since their performance is only slightly above chance. Apparently the amount of distortion involved in $50 \%$ Rc stimuli severely interferred with the attribute selection process.

The analysis of the shift in "D" bias from training to test showed that the lower the distortions in training, the greater the "D" bias in the test task. Somewhat unexpectedly, this bias is negatively related to performance in terms of S-D judgments. However, the notion of increased conservatism in setting requirements for class inclusion with training on increasingly undistorted stimuli is not without support. Posner (1968), in reviewing a number of studies, proposed that a major function of increased variability is in changing the criterion for acceptance of a new pattern as a member of the same category. He suggested that low variability training (high $\mathrm{Rc}$ ) aids $\mathrm{Ss}$ in becoming sensitive to the appropriate attributes and in abstracting a prototype, but leads to a conservative criterion for class inclusion. These results support Attneave's (1958) contention that Ss become sensitive to the variability of class members about their prototype as well as the appropriate set of attributes which define the family.

It is interesting to note that the group performances in these types of tasks, as in most learning experiments, may be misleading. The variances of the nine groups in the training and test phases are shown in Table 2; these variances are significantly heterogeneous in both tasks. The results of two Cochran tests show $\mathrm{C}(9,9)=.53$, $\mathrm{p}<.01$ in the training phase and $\mathrm{C}(9,9)=.31, \mathrm{p}<.01$ in the test phase. Variance appears to increase as $\mathrm{Rc}$ increases and to a lesser extent as ET increases in training. This variance reflects individual differences in S-D performance; in fact, at the high Rc level Ss' performance appears to be bimodal, while at the lower levels performance is considerably more homogeneous. Models of this behavior should reflect these individual differences.

In conclusion, the results of this experiment have the following implications for the issues cited in the introduction:

(1) Ss can, without feedback, select an appropriate set of attributes upon which to base their discrimination of stimuli produced as relatively complex distortions of various prototypes. This finding is an important prerequisite for the extension of the schema plus correction hypothesis to real-world pattern perception.
(2) Ss apparently become sensitive to the variability of family members about their prototype (i.e., the average amount of deviation) and make use of this information in making classification judgments.

(3) Unsupervised training on Markov prototypes enhances subsequent classification performance on distorted instances of the same schema families. This enhancement is greater than that which occurs with training on patterns at the same level of distortion as the test stimuli.

(4) Increased stimulus exposure time at high Rc (low distortion) tends to improve performance, presumably by allowing the Ss to respond to and encode a larger subset of the attributes relevant to stimulus classification. At low Rc (high distortion), the greater proportion of nonschematic information available in the patterns apparently inhibits improvement on the basis of increased viewing time.

\section{REFERENCES}

Attneave, F. Transfer of experience with a class-schema to identification-learning of patterns and shapes. Journal of Experimental Psychology, 1957, 54, 81-88.

Baker, R. A., \& Osgood, C. W. Discrimination transfer along a pitch continuum. J ournal of Experimental Psychology, 19.54, 48, 241-246.

Bersted, C. T., Brown, B. R., \& Evans, S. H. A standard set of Vargus 7 patterns at three levels of schematic redundancy. Psychonomic Monograph Supplements, 1968a, 2, 251-282.

Bersted, C. T., Brown, B. R., \& Evans, S. H. Transfer of schematic concept formation as a function of constraint redundancy and inter-task interval. Unpublished manuscript, Texas Christian University, 1968b.

Bregman, A. S. Analysis and classification of patterns by humans. Paper presented at McGill University Pattern Recognition Seminar, July 22-26, 1968.

Brown, B. R., \& Dansereau, D. F. Discrimination among schematic stimuli as a function of response mode, constraint redundaricy and form of Markov rule. Psychonomic Science, 1969, 17, 197-198.

Brown, B. R., \& Evans, S. H. Perceptual learning in pattern discrimination with two and three schema categories. Psychonomic Science, 1969, 15, 101-103.

Brown, B. R., Walker, D. W., \& Evans, S. H. Schematic concept formation as a function of constraint redundancy and knowledge of results. Psychonomic Science, 1968, 11, 75-76.

Evans, S. H. Vargus 7: Computed patterns from Markov processes. Behavioral Science, 1967a, 12, 323-328.

Evans, S. H. Redundancy as a variable in pattern perception. Psychological Bulletin, 1967b, 67, 104-113.

Evans, S. H., \& Mueller, M. R. Vargus 9: Computed stimuli for schema research. Psychonomic Science, 1966, 12, 511-512.

Harris, D. R., Dansereau, D. F., \& Hastings, M. A. The effect of temporal context on the classification of schematic patterns. Paper presented at Southern Society of Philosophy \& Psychology, Durham, N.C., March 1970.

Hastings, M. A. Feature selection: The effect of attribute variability on visual pattern recognition. Unpublished master's thesis, Texas Christian University, 1970.

Hastings, M. A., Dansereau, D. F., \& Dixon, T. R. Influence of pattern variance and overt responding on subjective categorization in a two-schema SCF task. Psychonomic Science, 1969, 16, 325-327.

Hinsey, W. C. Identification-learning after pretraining on central and non-central standards. Unpublished master's thesis, University of Oregon, 1963. 
Lawrence, D. H. The transfer of a discrimination along a continuum. Journal of Comparative \& Physiological Psychology, 1952, 45, 511-516.

Norcross, B. A study in concept formation. Unpublished master's thesis, McGill University, 1967.

Oldfield, R. C. Memory mechanisms and the theory of schemata. British Journal of Psychology, 1954, 45, 14-23.

Posner, M. I. Abstraction and the process of recognition. In J. T. Spence and G. H. Bower (Eds.), The psychology of learning and motivation. Vol. 3. New York: Academic Press, 1969.

Posner, M. I., \& Keele, S. W. On the genesis of abstract ideas. Journal of Experimental Psychology, 1968, 77, 353-363.

Posner, M. I., \& Keele, S. W. Retention of abstract ideas. Journal of Experimental Psychology, 1970, 83, 304-308.

Rankin, W. C., \& Evans, S. H. Facilitation of schematic concept formation as a function of two within-schema pretraining modes. Psychonomic Science, 1968, 13, 325-326.

Restle, F. A theory of discrimination learning. Psychological Review, 1955, 62, 11-19.

Vinikoor, D. A study in concept formation. Unpublished honors thesis, McGill University, 1968.

Woodworth, R. S. Experimental psychology. New York: Hoit, 1938.

(Received for publication July 14, 1973; accepted August 17, 1973.) 